\title{
Numerical simulation of a synthetic jet with OpenFOAM
}

\author{
Qiong Liu, Asimina Kazakidi, Marcello A.F.Medeiros and Vassilis Theofilis
}

\begin{abstract}
Numerical simulations of flow surrounding a synthetic jet actuating device are presented. By modifying a dynamic mesh technique available in OpenFOAM,a well-documented open-source solver for fluid dynamics, detailed computations of the sinusoidal motion of the synthetic jet diaphragm were possible. Numerical solutions were obtained by solving the two dimensional incompressible viscous N-S equations, with the use of a second order implicit time marching scheme and a central finite volume method for spatial discretization in both streamwise and crossflow directions. A systematic parameter study is reported here, in which the external Reynolds number, the diaphragm amplitude and frequency, and the slot dimensions are varied.
\end{abstract}

\section{Introduction}

Synthetic jets are commonly generated by the time-periodic ejection and suction of fluid across an orifice, resulting in zero-net mass flux [7]. Since the experimental work of Ingård and Labate [9] in the '50s, the study of synthetic jets has become one of the most attractive subjects of research in fluid mechanics. The primary reason behind is that the synthetic jet can be utilized as a flow control method, with several engineering applications [2], [7]. Originally demonstrated in the laboratory

Qiong Liu and Vassilis Theofilis

School of Aeronautics, Universidad politècnica de Madrid, Pza. Cardenal Cisneros 3, E-28040

Madrid Spain e-mail: liuqiong.upm@gmail.com

Asimina Kazakidi

Institute of Computer Science Foundation for Research \& Technology - Hellas (FORTH) GR70013

Heraklion, Greece

Marcello A.F.Medeiros

University of São Paulo. Escola de Engenharia de São Carlos. EESC 13566-590 São Carlos, SP, Brazil 
by Wiltse and Glezer [16], synthetic jets can further work as micro-fluidic devices with a potential implementation to thrust vectoring of jet engines.

A series of studies have been devoted to the control of synthetic jets in the past years. Chen et al. [4] and Davis and Glezer [6] have presented the mixing enhancement for the active control of separation and turbulence in the boundary layer (see also Smith and Glezer [15], and Crook et al. [5]). Mittal et al. [12] studied the performance of a synthetic jet actuator, based on a number of geometrical, structural and flow parameters. Amitay et al. [1] demonstrated the usage of synthetic jets in delaying separation. Further works on synthetic jets included two-dimensional simultions in quiescent flow conditions by Kral et al. [10] and Lee and Goldstein [11]. Moreover, Sahni et al. [13] have studied, both experimentally and numerically, the three-dimensional flow interactions between a finite-span synthetic jet and a crossflow, using a chord-based model.

The basic features of a synthetic jet actuator are illustrated schematically in Figure 1. Trains of vortices induced by the harmonic motion of a diaphragm located opposite to the slot, interact to produce the jet. The diaphragm amplitude and frequency, and the slot dimensions may change the characteristics of the jet's evolution. In addition, the jet flow may be significantly reshaped during the ejection and suction phases. It is critical therefore to characterize this evolution, in order to understand the flow physics of synthetic jets.

In the current study, we modified a dynamic mesh technique available in OpenFOAM, in order to conduct a series of numerical simulations of the synthetic jet model. The paper is organized as follows: Section 2 presents the numerical framework, Section 3 discusses the computational results, and Section 4 summarizes the results of this study.
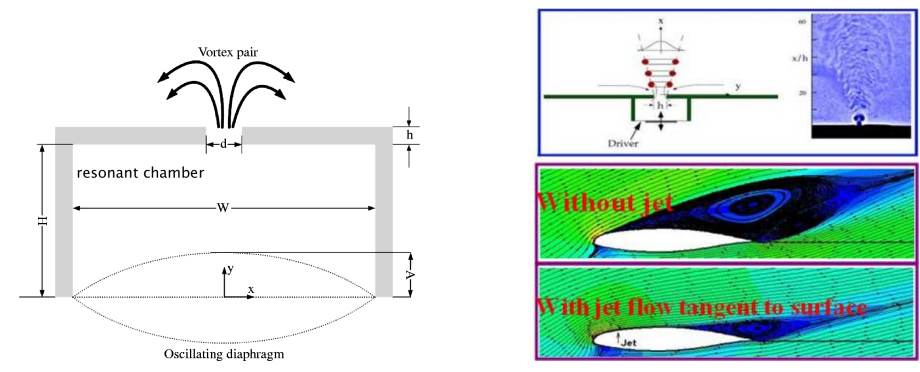

Fig. 1 (Left) Schematic diagram of a synthetic jet actuator [3]. (Right) Application of a jet actuator on an airfoil, with evident decrease of the downstream separated region [8].

\section{Methods}

\subsection{Actuator geometry and computational mesh}

The two-dimensional synthetic jet actuator considered in this study was comprised of a rectangular cavity, the upper wall of which included a thin slot of width $d$ and width-to-height ratio $(d / h)$ of 1 ; the lower wall opposite to the slot formed an oscillating diaphragm (cf. Figure 2). The cavity was $20 d$ in width and $4 d$ in 
height, connected via the slot to a rectangular space that was $d$ in width and $2 d$ in height, above the cavity. This geometry was used as a reference geometry upon which several geometrical and flow parameters were tested (see Section 2.3).

The computational mesh of the actuator geometry was constructed using the mesh generator available in the OpenFOAM library. A mesh of $107 \times 54$ quadrilateral elements was used for the overall computational domain, of which $30 \times 25$ elements were used inside the slot and $118 \times 58$ elements inside the rectangular cavity. A very fine boundary layer mesh was constructed near the walls to adequately capture the viscous flow effects.

\subsection{Governing equations and numerical tools}

The 2-D incompressible viscous Navier-Stokes equations are written in conservation form as follows, assuming unit fluid density:

$$
\begin{array}{r}
\nabla \cdot \mathbf{u}=0 \\
\frac{\partial \mathbf{u}}{\partial t}+(\mathbf{u} \cdot \nabla) \mathbf{u}=-\nabla P+v \nabla^{2} \mathbf{u}
\end{array}
$$

where $\mathbf{u}$ is the velocity vector, $P$ is the pressure and $v$ is the kinematic viscosity of the Newtonian fluid.

Numerical simulations were carried out with OpenFOAM, an open-source C++ library with a wide collection of processing applications. Solution of the governing equations was achieved with a second order implicit time marching scheme and a central finite volume method for spatial discretization.

The motion of the diaphragm was simulated by modifying a dynamic mesh technique available in OpenFOAM. The class dynamicInkJetFvMesh is modified here for generate the mesh. The mesh motion was controlled by changing the frequency and the amplitude of the diaphragm's forced oscillation. Figure 2 shows an example of the dynamic mesh displacement during the periodic movement of the diaphragm.
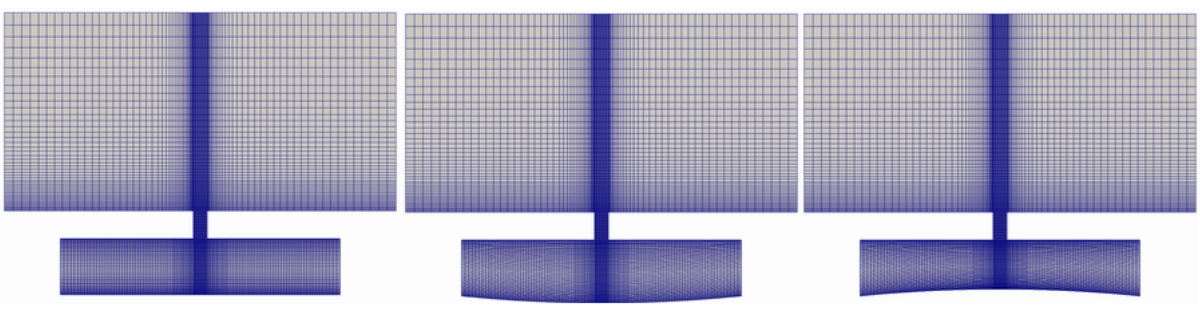

Fig. 2 Dynamic mesh displacement during the harmonic motion of the diaphragm in the synthetic jet actuator. 


\subsection{Boundary conditions and fluid parameters}

The diaphragm is modeled as a plate oscillating in its fundamental mode, according to the following motion profile:

$$
0.5 *(\cos (2 \pi t f)-1)
$$

The diaphragm has its maximum deflection at the center of the cavity and zero deflection at the two ends. Focusing on the parameters that are expected to have a strong influence on the characteristics of the synthetic jet. In the current study, we chose to systematically study the diaphragm amplitude $A / H$, the frequency $f$, the width-to-height ratio of the slot $(d / h)$, and the external flow Reynolds number $R e_{d}$, based on the slot width. The selected parameters are shown in Table 1

Assuming no external flow, the pressure gradient is zero. The Neumann and Dirichelet boundary conditions were used. For the cases where external flow is considered, the direction of flow is from let to right, according to Figure 2.

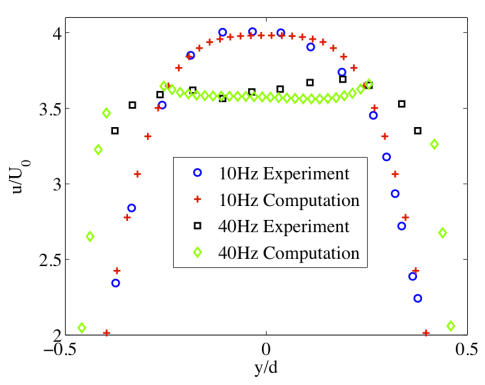

Fig. 3 Profile of velocity necessary for jet formation at $t / T=0.25$ for $d=0.0051 \mathrm{~m}$.

\begin{tabular}{c|c|c|c|c|c|c}
\hline \hline & $\mathrm{A}$ & $\mathrm{B}$ & $\mathrm{C}$ & $\mathrm{D}$ & $\mathrm{E}$ & $\mathrm{F}$ \\
\hline$R e_{d}$ & 0 & 0 & 0 & 0 & 71.426 & 250 \\
\hline $\mathrm{d} / \mathrm{h}$ & 1 & 1 & 0.5 & 1 & 1 & 1 \\
\hline $\mathrm{f}$ & 0.05 & 0.05 & 0.05 & 0.1 & 0.05 & 0.05 \\
\hline $\mathrm{A}$ & 0.1 & 0.2 & 0.2 & 0.2 & 0.2 & 0.1 \\
\hline Stokes Number & 3.32 & 3.32 & 3.32 & 4.7 & 3.32 & 3.32 \\
\hline \hline
\end{tabular}

Table 1 The values of frequency, amplitude and slot width-to-height ratio chosen for the presented parametric study.

\section{Results}

Velocity profiles at the peak of the blowing stroke $(t / T=0.25)$ are shown in Figure 3, which are good agreement with the experiment data [14]. Figure 4 shows sequences of instantaneous contour plots of spanwise vorticity, at four different stages of the diaphragm's oscillation cycle: at maximum expulsion, minimum volume, maximum ingestion and maximum volume. The first four cases (A to D) are simulated with no external flow, i.e. $R e_{d}=0$, whereas the last two cases (E to F) assume the presence of external flow. The parameters used for all numerical simulations are those shown in Table 1.

In case A, the diaphragm amplitude is small, and therefore vortices generated near the slot during each stage of the cycle, remain in the region without escaping the cavity. Specifically, during the expulsion stroke, a symmetric pair of counterrotating vortices is generated near the slot lips, in the direction above the cavity; during the ingestion stroke, a new pair of vortices is formed inside the cavity. Doubling the amplitude of the diaphragm oscillation (case B) resulted in vortices that 
escape the cavity, during the maximum expulsion stage, and travel inside the external space. These vortices do not interact further with the synthetic jet. During the maximum ingestion stage, fluid particles just above the slot are sucked back in the cavity and travel towards the transversely oscillating diaphragm plate. Flow impinging on the plate results in a layer of stagnant fluid. Increasing the height of the slot at twice the previous value, while maintaining the same oscillation amplitude (case C), had no significant influence on the evolution of the synthetic jet. Increase of the diaphragm frequency to the double value of that in case $\mathrm{B}$, while maintaining the same amplitude and slot width-to-height ratio (case D), had only a small influence on the evolution of the vortices, as compared to case B, with slightly greater sepa-

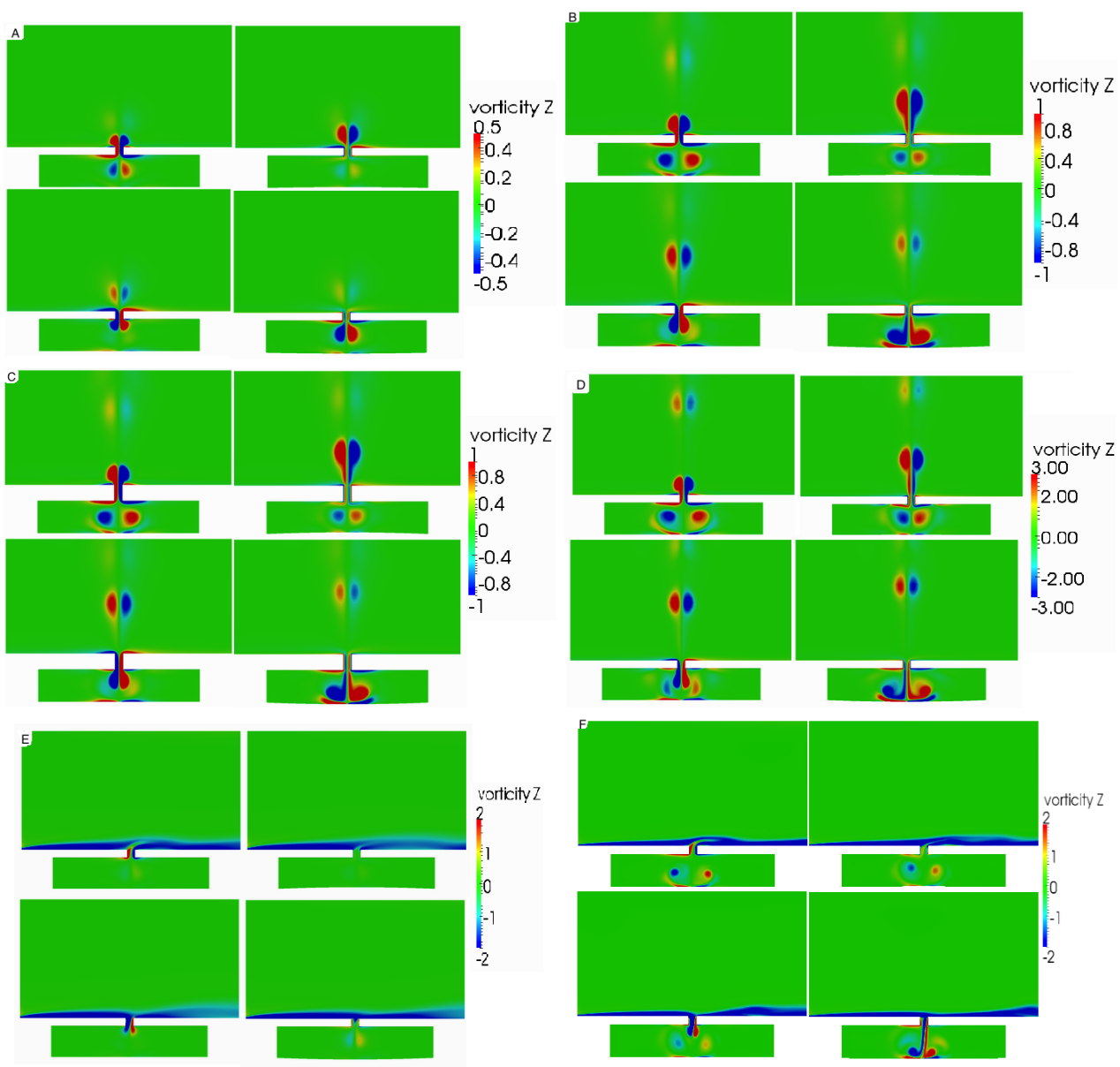

Fig. 4 Instantaneous contours of vorticity at four different stages of the diaphragm oscillation, in the absence (cases A to D) and in the presence (cases E and F) of external flow. The parameters used for the simulations are presented in Table 1. 
ration. In the final two cases $\mathrm{E}$ and $\mathrm{F}$, the presence of external flow drastically alters the evolution of the synthetic jet, which through interaction with the external flow, reshapes and becomes highly asymmetric within the cavity. The jet also affects the external flow at the regions downstream of the slot, by periodically altering the wall stresses.

\section{Conclusion}

A synthetic jet actuator has been parametrically studied here, by utilizing a modified dynamic mesh technique. The diaphragm is modeled to undergo harmonic oscillations. The evolution of the synthetic jet in the absence of external flow, showed that the amplitude and frequency have a stronger influence on the characteristics of the jet, than the slot height. In the presence of external flow, the synthetic jet is greatly varied. By using appropriate parameters, the jet can control the flow development downstream of the slot.

\section{References}

1. M. Amitay, A. Honohan, M. Trautman, and A. Glezer. Modification of the aerodynamic characteristic of bluff bodies using fluidic actuators. AIAA Aerospace Sciences Meeting \& Exhibit, 97-2004, 1997.

2. L.N. Cattafesta III and M. Sheplak. Actuators for active flow control. Annu. Rev. Fluid Mech., 43:247-272, 2011

3. B. Celik and F.O. Edis. Micro-scale synthetic-jet actuator flow simulation with characteristicbased-split method. Aircraft Engineering and Aerospace Technology, 81:239-246, 2009.

4. Y. Chen, S. Liang, K. Aung, A. Glezer, and J. Jagoda. Enhanced mixing in a simulated combustor using synthetic jet actuators. AIAA Aerospace Sciences Meeting \& Exhibit, 990449, 1999.

5. A. Crook, A.M. Sadri, and N.J. Wood. The development and implementation of synthetic jets for the control of separated flow. AIAA Aerospace Sciences Meeting \& Exhibit, 99-3176, 1999.

6. S.A. Davis and A. Glezer. Mixing control of fuel jets using synthetic jet technology:velocity field measurement. AIAA Aerospace Sciences Meeting \& Exhibit, 99-0447, 1999.

7. A. Glezer and M. Amitay. Synthetic jets. Annu. Rev. Fluid Mech., 34:503-529, 2002.

8. http://hangkong.nwpu.edu.cn/home/Department/dptshow/DepartmentxofxHydromechanics.htm.

9. U. Ingård and S. Labate. Acoustic circulation effects and the nonlinear impedance of orifices. J Acoust Soc Am, 22(2):211-218, 1950.

10. L.D. Kral, J.F. Donovan, A.B. Cain, and A.W. Cary. Numerical simulation of synthetic jet actuators. AIAA Aerospace Sciences Meeting \& Exhibit, 97-1824, 1997.

11. C.Y. Lee and D.B. Goldstein. Two dimensional synthetic jet simulation. AIAA J., 40:510-516, 2002.

12. R. Mittal, P. Rampunggoon, and H.S. Udaykumar. Interaction of a synthetic jet with a flat plate boundary layer. AIAA Aerospace Sciences Meeting \& Exhibit, 2773, 2001.

13. O. Sahni, J. Wood, K.E. Jansen, and M. Amitay. Three-dimensinal interactions between a finite-span synthetic jet and a crossflow. J. Fluid Mech., 671:254-287, 2011.

14. Barton L. Smith and Gregory W. Swift. Synthetic jet at large reynolds number and comparison to contimuous jets. AIAA Aerospace Sciences Meeting \& Exhibit, 3030, 2001.

15. B.L. Smith and A. Glezer. Vectoring and small-scale motions effected in free shear flows using synthetic jet actuators. AIAA Aerospace Sciences Meeting \& Exhibit, 97-0213, 1997.

16. J. Wiltse and A. Glezer. Manipulation of free shear flows using piezoelectric actuators. $J$. Fluid Mech., 249:261-285, 1993. 